\title{
TERAPIAS ALTERNATIVAS / COMPLEMENTARES \\ A VISÃO DO GRADUANDO DE ENFERMAGEM
}

\section{ALTERNATIVE PRACTICE OF HEALTH - THE VIEW OF NURSING STUDENTS}

\author{
TERAPIAS ALTERNATIVAS / COMPLEMENTARIAS - \\ LA VISION DE ALUMNO DE PRE-GRADO DE ENFERMERÍA
}

Monica Martins Trovó*

Maria elúlia Paes da Silva**

Trovó MM, Silva MJP. Terapias alternativas / complementares - a visão do graduando de enfermagem. Rev Esc Enferm USP 2002; 36(1): 80-7.

\section{RESUMO}

O objetivo desta pesquisa foi verificar se os graduandos de Enfermagem conhecem Terapias Alternativas/Complementares (TA), se esse conhecimento é adquirido, durante a graduação, e se utilizam ou recomendam seu uso.Os dados foram coletados de 96 alunos, dos 4 anos de graduação distribuidos eqüitativamente, de maneira que equivalessem a 30\% dos alunos de cada ano. Verificou-se que 93,73\% conhecem as T.A., que tal conhecimento não é adquirido durante a graduação, mas principalmente por meio do senso popular e que, embora a maioria (86,46\%) recomende seu uso, sobretudo por acreditar na sua eficácia, apenas cerca de um terço as utilizam.

PALAVRAS -CHAVE: Terapias alternativas. Terapias complementares. Enfermagem holística.

\begin{abstract}
The objective of this research was verify if Nursing students know Alternative Practices of Health (A.PH.), if-this knowledge is acquired during the graduation and if they use themselves or recommends this use. The bases were collected near to 96 students of the 4 years of graduation, that were shared out so that is the same as 30\% of the students. We verified that 93,73\% of the students know A.P.H., that this knowledge is not acquired during the graduation, but principally through the popular sense. Although the most of students $(86,46 \%)$ recommend the use of A.P.H., principally because they believe in its effectiveness, only about the third part makes use of them.
\end{abstract}

KEYWORDS; Alternative therapies. Complementry therapies. Holistic nursing.

\section{RESUMEN}

El objetivo de esta investigación fue verificar si los alumnos de pre-grado de enfermeria, conocen las Terapias Alternativas Complementarias (TA.), si ese conocimiento es adquirido durante el pre-grado y si utilizan y recomiendan su uso. Los datos fueron recolectados con 96 alumnos de los cuatro años de pré-grado en enfermeria, distribuidos equitativamente, de manera que equivalen al 30\% de los alumnos de cada año. Se verifico que 93,73\% conocen las T.A., que este conocimiento no es adquirido durante el pre-grado, sino principalmente por medio de censo popular, y aunque la mayoria (86,46\%) recomiende su uso, principalmente por creer en su eficacia, solo un tercio las utilizan.

PALABRAS -CLAVE: Terapias alternativas. Terapias complementarias. Enfermerfa holística.

Graduanda do sexto semestre da Escola de Enfermagem da Universidade de São Paulo. Bolsista do CNPq. -mail: trovomonica@ig.com.br

** Enfermeira. Professor Livre Docente do Departamento de Enfermagem Médico-Cirúrgica da Escola de Enfermagem da Universidade de São Paulo. E-mail: juliaps@usp.br 


\section{INTRODUÇÃO}

A enfermidade humana surgiu com a aparição do homem sobre a terra, assim como a necessidade de combatê-la(1).

É comprovado que doenças que ainda hoje afetam a humanidade, levando até a morte, têm as características descritas por antigas civilizações como, por exemplo, os egipcios, há dezenas de séculos atrás.

De acordo com Landmann (2) Hipócrates, o pai da Medicina, definia saúde como o estado de harmonia do homem com a natureza, o equilíbrio entre os diferentes componentes do organismo entre si e com o meio ambiente. Saúde e doença dependiam de uma perfeita interação da mente com o corpo e do homem com o meio onde ele vivia.

Na Idade Média, com a concepção da Igreja de que Deus era o responsável pela ordem natural e social e que a doença era um castigo pelos pecados cometidos, o conceito hipocrático de saúde e doença foi esquecido e ao médico foi limitado o cuidado do corpo, para aliviar o sofrimento.

O Renascimento foi acompanhado pelo crescente desenvolvimento da ciência e seguido pelo crescimento da cultura ocidental. A concepção hipocrática, antes deixada de lado, foi profundamente abalada com o paradigma mecanicista de Descartes, que criava uma rigorosa dicotomia entre corpo e mente(3)

O corpo, visto então como máquina, passou a ser tratado em partes cada vez menores. A doença, de acordo com essa concepção, era causada por defeitos das peças da máquina humana. À mente coube um papel irrelevante, um fantasma abrigado dentro do corpo máquina. Todas as funções do corpo dependiam do funcionamento independente do órgão(2).

O paradigma cartesiano é o responsável pelas bases da medicina científica e pela criação das especialidades médicas. No século XX, com as novas descobertas no campo da física, como as teorias de Einstein, abre-se um novo horizonte: a matéria é vista como manifestação de energia (4) $\mathrm{e}$, assim sendo, o ser humano, também matéria, é constituído de sistemas energéticos que interagem, formando um todo que deve ser equilibrado e harmonioso.

Segundo essa concepção, nós somos seres multidimensionais de energia, cujo corpo físico é apenas um dos componentes de um sistema dinâmico maior, ou seja, o homem é um complexo mente/corpo/ espírito, que existe em equilíbrio dinâmico contínuo com as dimensões energéticas superiores.

Nogueira(5) afirma que os principais componentes dessa visão holística são:

1) As entidades ou sistemas do universo existem como um todo, um conjunto, uma totalidade - nós fazemos parte de um todo, o universo.
2) As partes do conjunto ou unidade são dinamicamente independentes e inter-relacionadas somos únicos, desenvolvemos diferentes doenças que causam mudanças em determinadas partes, afetando as outras partes e, conseqüentemente, o todo.

3) A unidade não pode ser entendida pelo exame isolado das partes - quando separadas, não permitem sua real compreensão por continuar sendo parte de algo maior.

4) O todo é maior que a soma das partes - quando inter-relacionadas, as partes adquirem características e qualidades novas, inexistentes anteriormente, maiores que se somadas individualmente.

A compreensão da ação das Terapias Alternativas/Complementares é intimamente dependente da visão holográfica(1).

Sob a denominação de Terapias Alternativas/ Complementares entende-se as técnicas que visam a assistência de saúde ao indivíduo, seja na prevenção, seja no tratamento, considerando-o como um todo corpo/mente/espírito - e não como um conjunto de órgãos ou partes isoladas(6), diferentemente da assistência alopática ou medicina ocidental, cujo objetivo é a cura da doença pela intervenção direta no órgão ou parte doente.

Segundo Gerber( ${ }^{(4)}$ o que atualmente é considerado boa saúde é um estado de neutralidade, de "ausência de sintomas", em que não existem problemas identificáveis. O estado de neutralidade é diferente de bem-estar, que é o objetivo da medicina holística, para a qual o homem encontra-se em um estado ótimo de integração entre os elementos do corpo, da mente e do espírito.

A doença é sempre uma pausa, uma interrupção nos padrões de hábitos nos quais vivemos durante muitos $\operatorname{anos}^{(7)}$ Ela pode despertar, em algumas pessoas, a necessidade de compreender mais profundamente quem elas são e o que é mais importante para elas, pode levar outras a viverem mais conscientemente, valorizando mais as coisas habituais, como por exemplo, as emoções que as influenciam.

De acordo com Silva; Benko(8), temos participação ativa no processo saúde-doença e especialmente no de cura e, por muitas vezes, tentamos separar o corpo de nossas emoções, buscando explicação de nossos males em causas externas. As enfermidades não provêm necessariamente do meio exterior; muitas vezes, elas se originam em nossa própria estrutura fisica, dentro de nós.

A chave do tratamento das doenças depende de nossa capacidade de compreender como elas se manifestam. Assim sendo, procuramos tratamentos complexos e dispendiosos para doenças que possuem componentes psicológicos e poderiam ser perfeitamente resolvidas com o desenvolvimento do autoconhecimento, assim como o conhecimento de técnicas 
complementares, que aliadas ou não ao tratamento alopático, podem melhorar significativamente a qualidade de vida do indivíduo.

Souza; Silva(1), citando Nogueira (5), afirmam que, sob o ponto de vista holístico, ao prestar assistência à saúde, o profissional visa harmonizar e equilibrar entre si todas as dimensões do ser humano, atuando não só no corpo físico, mas também nas energias mais sutis que formam seu corpo. Para tanto, há necessidade de buscar-se formas alternativas de assistência, que tratem do corpo, da mente e do espírito, sem contudo haver necessidade de lidar diretamente com crenças religiosas.

Barbosa(9), assim como Hill(6), agrupa as Terapias Alternativas / Complementares em:

- Terapias fisicas: acupuntura, moxabustão, shiatsu (e outras massagens), do-in, argiloterapia, cristais.

- Hidroterapia: hidroterapia (não especificada), banhos, vaporização e sauna.

- Fitoterapia: fitoterapia (não especificada), ervas medicinais, florais.

- Nutrição: nutrição alternativa (não especificada), terapêutica nutricional ortomolecular.

- Ondas, radiações e vibrações: radiestesia, radiônica.

- Terapias mentais e espirituais: meditação, relaxamento psicomuscular, cromoterapia, toque terapêutico, visualização, Reich.

- Terapia de exercícios individuais: biodança, vitalização.

Convém lembrar que a Organização Mundial de Saúde (OMS) agrupa essas terapias alternativas sob a denominação de medicina tradicional (10) visto que, na cultura oriental, elas são conhecidas e aplicadas há séculos.

A população mundial cresceu em ritmo acelerado, nas últimas décadas, e, com ela, as cidades, as condições subumanas de sobrevivência e a carência por um sistema de saúde eficaz. Dados da $\mathrm{OMS}^{(10)}$ mostram que, já na década de 70, para dois terços da população mundial, as técnicas alternativas/complementares eram ainda a única fonte de assistência à saúde. Estudos recentes mostram que a prevalência do uso de Terapias Alternativas/Complementares, em 12 grandes cidades de diferentes continentes varia de 9 a 65\%(11)

Existe, no mundo todo, crescente interesse por este tipo de tratamento. A Folha de São Paulo, em 11/ 05/00, apresentou um encarte denominado "Folha Equilibrio", abordando as Terapias Alternativas/ Complementares já utilizadas em três grandes centros de saúde da cidade de São Paulo (musicoterapia, relaxamento/yoga e acupuntura) (12).
Segundo Price(13), perfumes e óleos de aroma-terapia são comprovadamente úteis no controle do mau cheiro causado pelas feridas em pacientes oncológicos. Com o controle do odor, pode-se inclusive controlar melhor a dor, visto que esta possui componentes emocionais e vários fatores podem agravá-la, como por exemplo, a baixa auto-estima do paciente.

O crescente interesse pelo uso de Terapias Alternativas/Complementares na assistência à saúde deve-se, segundo $\operatorname{Nogueira}^{(14)}$ a vários fatores, tais como:

- preço elevado da assistência médica privada, associado ao alto custo dos medicamentos, além da precariedade da assistência prestada pelos serviços públicos em geral;

- verifica-se, na maioria das vezes que as terapias alternativas são tão eficazes como a terapêutica "científica" / aloterapia, além do que, se corretamente utilizadas, não ocasionam efeitos colaterais danosos ao organismo, embora seu mecanismo não esteja totalmente esclarecido;

- há um certo ressentimento com a ciência oficial, visto que ela não consegue realizar o sonho de felicidade do ser humano.

A enfermeira exerce papel fundamental nesse contexto: é ela que, em virtude de seu trabalho, está em contato direto e mais profundo com a população, seja em centros de saúde, hospitais, seja na comunidade, tendo a oportunidade de educar e esclarecer a população quanto ao uso nocivo ou benéfico dessas técnicas ${ }^{(15)}$

Calixto(16) afirma que a idéia de que drogas naturais são seguras e livres de efeitos colaterais é falsa. As plantas contêm centenas de constituintes e alguns deles são tóxicos, podendo prejudicar o organismo daqueles que buscam auxilio no tratamento de seus males. Em um país como o Brasil, onde são vendidas "garrafadas" e balconistas de farmácia prescrevem potentes antibióticos, o papel elucidativo do enfermeiro é fundamental, visto que ele possui o conhecimento técnico-científico e habilitação para tanto.

O Conselho Federal de Enfermagem (COFEN), no Parecer Informativo 004/95(17), reconhece a fundamentação da profissão de Enfermagem, na visão holística do ser humano, o crescente interesse e utilização das práticas naturais no cuidado ao cliente e os aspectos do Código de Ética dos Profissionais de Enfermagem que permitem a utilização das terapias naturais. Finalmente, em 19/3/1997, o COFEN, por meio da Resolução 197 "Estabelece e reconhece as Terapias Alternativas como especialidade e/ou qualificação do profissional de Enfermagem" (18). 
Em outras palavras, o enfermeiro, que antes tinha apenas cursos extracurriculares ou disciplinas optativas sobre Práticas Alternativas/ Complementares, durante seu curso de graduação, ou ainda apenas conhecimentos herdados da cultura popular, agora pode ser identificado como especialista em Práticas Alternativas, mediante conclusão e aprovação em cursos reconhecidos em instituição de ensino, com carga horária mínima de 360 horas $^{(8)}$

Em recente estudo realizado, no Canadá, com alunos do último ano dos cursos de graduação em Medicina, Farmácia, Fisioterapia, Terapia Ocupacional e Enfermagem, sobre conhecimento e opinião a respeito de técnicas alternativas/complementares, os resultados mostram que são os futuros enfermeiros os que mais fazem uso de tais técnicas $(44,7 \%)$, comparado com $18,2 \%$ dos estudantes de Farmácia e '10,0\% de Medicina. Com relação ao conhecimento das técnicas, os dados da pesquisa mostram que varia de acordo com a área do futuro profissional, sendo que os estudantes de Farmácia mostraram mais conhecimento sobre a medicina herbária e a homeopatia, os de Fisioterapia apreciam a acupuntura e as massagens terapêuticas enquanto os de Enfermagem conhecem mais o toque terapêutico(19)

Segundo Barbosa ${ }^{(9)}$, no Brasil, 47 enfermeiros já utilizavam as Terapias Alternativas / Complementares, no tratamento de seus pacientes, em 1993, antes mesmo de seu reconhecimento como especialista.

Em estudo realizado por Silva; Benko(8), 66,1\% dos enfermeiros docentes por elas entrevistados, em quatro instituições de ensino de graduação em Enfermagem da cidade de São Paulo fazem uso das Terapias Alternativas/Complementares, embora, dentre eles, apenas 58,9\% abordem o assunto com seus alunos.

Reed et $\mathrm{al}^{(20)}$ apontam várias razões para os educadores em Enfermagem incluírem o ensino de Terapias Alternativas/Complementares, no planejamento das experiências do estudante, entre elas, o fato de que, para muitos pacientes, o cuidado de Enfermagem traz perspectivas e seleciona tentativas de cura que não estão de acordo com as crenças e práticas da medicina científica. Além disso, o educador em Enfermagem deve ter como meta educacional o desenvolvimento da sensibilidade cultural no aluno, com informações que possam modelar as atitudes e o comportamento relatados na saúde e na doença.

Visto que, durante o período de graduação em Enfermagem é que se adquire os conhecimentos básicos necessários sobre o processo saúde - doença e se desenvolve o interesse pela futura área de atuação como, por exemplo, Obstetrícia ou Práticas
Alternativas/Complementares?, surgem as questões: será que o graduando de hoje conhece as Práticas

lternativas/Complementares? Será que sabe de sua regulamentação como especialidade para o enfermeiro? Será que ele faz uso? E, conhecedor dessas terapias, saberia aplicá-las em seus pacientes?

\section{OBJETIVOS DO ESTUDO}

Diante da problemática exposta, temos como objetivos, neste trabalho:

- Verificar se os graduandos de Enfermagem conhecem as Práticas Alternativas/Complementares.

- Verificar se esse conhecimento é adquirido, durante a graduação, e em que período.

- Verificar se estes graduandos utilizam e/ou recomendam o uso das Práticas Alternativas/ Complementares e $\quad$ o porquê.

\section{MATERIAL E MÉTODO}

- Tipo de estudo: trata-se de um estudo exploratório, descritivo, transversal e de campo.

- Local de estudo: o estudo foi realizado na Escola de Enfermagem da Universidade de São Paulo (EEUSP).

- População: foram entrevistados 30\% dos alunos regularmente matriculados no curso de graduação, sendo o número total, 321 alunos, distribuído eqüitativamente entre os 4 anos de graduação. Assim sendo, foram entrevistados 28 alunos do primeiro ano, 22 alunos do segundo ano, 26 alunos do terceiro ano e, finalmente, 20 alunos do quarto ano do curso de graduação em Enfermagem da EEUSP perfazendo um total de 96 alunos.

- Procedimento de coleta de dados: após a aprovação pelo Comitê de Ética da Escola de Enfermagem da Universidade de São Paulo, a coleta de dados se deu no período de agosto e setembro de 2.000 , mediante questionário aplicado ao aluno, com prévia autorização escrita do mesmo (consentimento livre e esclarecido). Os alunos foram informados sobre o anonimato na utilização dos dados para a pesquisa e que, qualquer dúvida sobre o presente estudo, poderia ser esclarecida com a aluna bolsista ou a docente orientadora.

Os alunos dos quatro anos de graduação foram aleatoriamente escolhidos para a coleta de dados, obedecendo apenas o critério da abordagem oportuna, sempre no intervalo das aulas. De modo geral, a coleta de dados transcorreu de forma tranqüila. Os alunos, sem exceção, mostraram-se solícitos, respondendo prontamente ao questionário no momento da abordagem. 
Concomitantemente à coleta de dados, foi realizado o levantamento bibliográfico para atualização do estudo, utilizando-se dados do MEDLINE, referente ao período de cinco anos (1995-2000). Ao pesquisar as key-words alternative practice/nursing/students, foram encontrados 18 títulos, dos quais apenas 4 foram utilizados, já que os demais ou não estavam diretamente relacionados com o tema do presente estudo, ou não estavam disponiveis para consulta no Brasil. Tomandose como base o mesmo banco de dados, no mesmo período, foi realizada nova pesquisa, desta vez utilizando-se como key-words holism/nursing. Esse levantamento resultou em 67 referências publicadas, das quais 11 foram selecionadas e apenas 3 estavam disponiveis para consulta.

- Tratamento dos dados: os dados foram analisados por número e porcentagem. Usamos a classificação proposta por Hill (6) e mantida no trabalho de pesquisa de Barbosa (9) para a categorização das práticas alternativas/comple-mentares citadas pelos alunos.

\section{APRESENTAÇÃO OS RESULTADOS DISCUSSÃO}

Dos 96 (100\%) graduandos entrevistados, 88 $(91,67 \%)$ são do sexo feminino e $8(8,33 \%)$ masculino. A média de idade dos entrevistados é de 21,5 anos. Podemos perceber que a população estudada é composta predominantemente de mulheres jovens. Quando questionados sobre com quem moram, 86 $(89,59 \%)$ dos entrevistados afirmaram morar com a família (entendemos por familia, os pais, avós, irmãos, tios e primos), $7(7,29 \%)$ referiram morar com amigos e apenas $1(1,04 \%)$, sozinho.

Quando questionados se possuíam crença religiosa, $\quad 83 \quad(86,46 \%) \quad$ responderam afirmativamente, $11(11,46 \%)$ referiram não possuir e $2(2,08 \%)$ não responderam. Com relação ao tipo de crença religiosa da população estudada, os resultados são apresentados na Tabela 1.

Tabela 1: Crença religiosa referida pelos graduandos de Enfermagem. São Paulo, 2000.

\begin{tabular}{lcc}
\hline Crença & númer & porcentage \\
\hline Catolicismo & 3 & 37,5 \\
Espiritismo & 1 & 16,6 \\
Cristianismo & 1 & 13,5 \\
Não segue religião, mas & & 8,34 \\
acredita & & 4,17 \\
em Deus & & 2,08 \\
Catolicismo e Espiritismo & & 1,04 \\
Elementos de várias religiões & & 1,04 \\
Seicho-no-ie & 1 & 1,04 \\
Budismo & & 1,04 \\
Candomblé & & 13,5 \\
Orixás & 9 & 100,0 \\
\hline Total & & \\
\hline
\end{tabular}

Podemos verificar, pela Tabela 1 , que a maioria dos graduandos segue a doutrina católica; dado esperado por ser o País predominantemente católico. É interessante notar que $13,54 \%$ dos entrevistados consideram o cristianismo crença religiosa, não seguindo uma religião, especificamente, mas os preceitos das religiões cristãs.

Tabela 2: Relação das terapias alternativas/ complementares conhecidas por alunos de graduação em Enfermagem da EEUSP. São Paulo, 2000.

\begin{tabular}{lcc}
\hline $\begin{array}{l}\text { Grupos e tipos de terapias } \\
\text { citadas }\end{array}$ & númer & porcentage \\
\hline fisioterapia & & \\
\hline 1.Florais & 5 & 60,4 \\
2.Fitoterapia & 1 & 17,7 \\
3.Aromaterapia & - & 5,21 \\
4.Medicina herbária & - & 2,08 \\
\hline Terapias fisicas & & \\
\hline 1.Acupuntura & 5 & 53,1 \\
2.Massagem (não especificada) & 2 & 23,6 \\
3.Do-in & 1 & 12,5 \\
4.Moxabustão & & 3,21 \\
5. Shiatsu & & 3,21 \\
Concepção Globais & & \\
1.Homeopatia & 4 & 44,8 \\
\hline
\end{tabular}

Terapias mentais e espirituais

\begin{tabular}{lll}
\hline 1.Cromoterapia & 2 & 27,7 \\
2.Musicoterapia & 2 & 20,8 \\
3.Toque terapeutico & & 5,21 \\
4.Reich & 5,21 \\
5.Meditação & 1,04 \\
6.Cirurgia Psiquica & 1,04 \\
7.Hipnose & 1,04 \\
\hline Hidroterapia & \\
\hline
\end{tabular}

\begin{tabular}{|c|c|}
\hline 1.Hidroterapia (não especificada) & 3,12 \\
\hline \multicolumn{2}{|l|}{ Nutrição } \\
\hline $\begin{array}{l}\text { 1.Terapia nutriciona orto } \\
\text { molecular }\end{array}$ & 3,13 \\
\hline 2.Nutrição não especificada & 2,08 \\
\hline \multicolumn{2}{|l|}{ Ondas, Radiações e Vibrações } \\
\hline 1.Radiestia & 2,08 \\
\hline \multicolumn{2}{|l|}{ Terapias exerc[icios individuais } \\
\hline 1.Yoga & 2,08 \\
\hline \multicolumn{2}{|l|}{ Método de Diagnose } \\
\hline 1.Iridologia & 1,04 \\
\hline outros & 1,04 \\
\hline
\end{tabular}

*Usaremos o total de citações feitas pois temos entrevistados que citam mais de uma prática, modo de conhecimento ou tipo de resultado observado. 
Ao serem questionados se conheciam alguma Terapia Alternativa/Complementar (T.A.), 90 (93,75\%) dos entrevistados afirmaram que sim, $6(6,25 \%)$ referiram não conhecer e $1(1,04 \%)$ não respondeu. Dentre as T.A. conhecidas pelos graduandos, a mais citada* $^{*}$ foi a terapia floral (58 vezes, ou seja, 60,42\%). Em seguida, vêm acupuntura (51 citações), homeopatia(43), cromoterapia(26), massagem não especificada(23), musicoterapia(20), fitoterapia(17) e do-in(12). Aromaterapia, toque terapêutico e reich foram citados 5 vezes cada. Tivemos ainda dez citações de práticas que não obedecem à classificação proposta por Hill (6), como por exemplo, medicina natural, cirurgia espiritual, eqüinoterapia.

Pela análise dos dados da Tabela 2, percebemos que a maioria dos entrevistados conhece a terapia floral, dado que coincide com o encontrado por Barbosa(9), há 6 anos, quando desenvolveu sua pesquisa com enfermeiros de todo o Brasil e com o trabalho de Silva; Benko(8), com os docentes de escolas de Enfermagem de São Paulo.

Questionados sobre o modo como ficaram conhecendo as T.A., os graduandos referiram, com maior freqüência (33 vezes, o que significa $34,37 \%$ dos graduandos entrevistados) livros, revistas e televisão. Vinte e sete graduandos $(28,12 \%)$ referiram amigos e familiares; $21(21,87 \%)$, pessoas que as utilizavam; $14(14,58 \%)$, a disciplina optativa "Enfermagem nas Práticas Complementares de Saúde", oferecida pela EEUSP; 10 (10,42\%), a EEUSP, não especificando se, durante uma disciplina ou não. Sete $(7,29 \%)$ afirmam conhecer as T.A. pois as utilizam ou já haviam utilizado. Foram ainda citados: docentes, médicos e Hospital Universitário (4 vezes cada), porque conhecem algum terapeuta ( 3 vezes), por meio de artes marciais, no centro espírita (com 1 citação cada). Não responderam à questão 6 graduandos $(6,25 \%)$.

Concluímos, pela análise dos dados descritos, que as noções do graduando sobre as T.A. se dá principalmente pelos veículos de comunicação e pelo conhecimento do senso comum, seja por intermédio de pessoas que as utilizam, seja por meio de informações transmitidas verbalmente, não fazendo parte do "saber oficial" do aluno. Nogueira (14) já afirmava tratar-se de conhecimento da população, adquirido de maneira informal, que faz parte da cultura de um povo, não podendo ser ignorado.

Com relação ao uso das T.A. pelos graduandos, $56(58,33 \%)$ referem não fazê-lo, enquanto 35 $(36,46 \%)$, o fazem. Quatro $(4,17 \%)$ entrevistados citaram não fazer uso atualmente, mas já o terem feito $\mathrm{e}$ $1(1,04 \%)$ refere não fazer uso, mas que gostaria de fazê-lo. Dados semelhantes foram encontrados no estudo de Baugniet et al (19), em que $44,7 \%$ dos graduandos de Enfermagem entrevistados fazem uso de técnicas alternativas.
É interessante notar que, embora a maioria $(93,75 \%)$ dos graduandos conheça pelo menos uma T.A., apenas aproximadamente um terço $(36,45 \%)$ as utiliza. Esses dados coincidem com os achados de Silva; Benko(8), em que embora $94,91 \%$ dos docentes por elas entrevistados afirmassem ser favoráveis ao ensino de T.A., apenas $66,1 \%$ as utilizavam.

Das terapias utilizadas pelos 35 alunos que responderam-fazer uso de alguma delas,.., a mais citada (16 vezes, $45,71 \%$ ) foi a floral, seguida de homeopatia (13 citações, $37,14 \%)$. A fitoterapia foi citada por $7(20,0 \%)$ dos graduandos, a massagem e a acupuntura por 4 $(11,43 \%)$; o do-in e a moxabustão foram citadas $3(8,57 \%)$ vezes cada e a cromoterapia 2 vezes $(5,71 \%)$. Foram referidas apenas 1 vez $(2,86 \%)$ cada: radiestesia, shiatsu, nutrição, Reich, yoga e RPG (Reeducação Postural Global). É importante referir que, durante o "Curso de Especialização em Terapia Floral", que ocorre na EEUSP os alunos podem se inscrever para serem atendidos pelos especializandos, acompanhados de terapeutas.

Os alunos foram ainda questionados se já haviam discutido sobre T.A. com docentes da EEUSP: $68(70,83 \%)$ responderam negativamente, enquanto 28 $(29,17 \%)$ positivamente. Dos 28, 13 (46,43\%) afirmaram ter discutido várias vezes; 4 (14,29\%), de 3 a 5 vezes; $3(10,71 \%), 2$ vezes e $3(10,71 \%), 1$ vez. Podemos verificar que, apesar de ser uma possibilidade de Especialização, as T.A. ainda não são discutidas com os alunos.

Dos 28 entrevistados que responderam em qual disciplina discutiram essa questão, 20 (71,43\%) citaram "Enfermagem nas Práticas Complementares de Saúde"; 4 (14,29\%) referiram conversas particulares com docentes.. As disciplinas "Fundamentação do Processo de Cuidar" e "Saúde do Adulto I" tiveram 3 (9,37\%) citações cada e "Saúde da Mulher", "Saúde da Criança" e "Administração" tiveram, cada uma, apenas $1(3,12 \%)$ citação. Foram ainda citadas, 1 vez cada, aulas de dor, consultas de florais e todas as disciplinas.

Os graduandos foram também questionados se haviam observado resultados com o uso de T.A., não sendo especificado se pelo próprio aluno ou por outras pessoas. Dos 96 entrevistados, 41 (42,71\%) afirmaram ter observado resultado, enquanto $18(18,75 \%)$ referiram não ter observado. Com relação ao resultado observado, 19 (46,34\%) citaram melhora da saúde/sintomas; 9 $(21,95 \%)$, alívio de dores; $8(19,51 \%)$, relaxamento; 6 $(14,63 \%)$, melhora de desânimo/depressão e tensão/ ansiedade. O aumento de resistência a doenças e a melhora do estado psicoespiritual foram citadas 2 vezes $(4,88 \%)$ cada. Tiveram ainda $1(2,44 \%)$ citação cada: aumento do autocontrole, sono tranqüilo, equilíbrio, regulação fisiológica, mudança de hábitos, boa aceitação do tratamento e aumento da compreensão sobre o assunto. 
Dos 96 graduandos, $83(86,46 \%)$ recomendariam o uso de T.A., enquanto $11(11,46 \%)$ referiram não recomendar e $2(2,08 \%)$ não responderam. Podemos notar que, embora muitos graduandos $(58,33 \%)$ não façam uso de T.A., a grande maioria as recomenda.

Entre os graduandos que recomendam o uso de T.A., $43(51,81 \%)$ justificam a indicação por acreditar na sua eficácia e ter vivenciado bons resultados com seu uso; $10(12,05 \%)$ referiram indicar como complemento à alopatia; $5(6,02 \%)$, por acreditar na importância de tratar o paciente como um todo; $3(3,61 \%)$, por considerarem menos agressivas ao paciente do que muitos tratamentos alopáticos; $2(2,41 \%)$ citaram a indicação de terapias específicas (acupuntura e massagem) e 8 $(9,64 \%)$, embora recomendem seu uso, não justificaram o motivo da indicação. Outras 12 indicações foram justificadas com resposta única: "o paciente melhora em menor tempo", "é um modo diferente de se buscar a cura", "reduz o stress e ansiedade", entre outras.

Questionados se o enfermeiro pode ser especialista em T.A., os graduandos foram unânimes: $92(95,83 \%)$ responderam afirmativamente, enquanto apenas 4 deixaram a questão em branco.

Quando solicitados a justificar o motivo de o enfermeiro poder ser especialista em T.A., 17 (18,48\%) não souberam responder, $16(17,39 \%)$ referiram ser possível a especialização, pois o enfermeiro pode estudar e "aprofundar-se" no tema. Apenas 8 (8,70\%) graduandos apontaram ser possivel a especialização por seu caráter legal e a existência de cursos registrados para tal formação. O restante respondeu valorizando as T.A. ou reafirmando saber seu crescimento na atualidade.

\section{CONCLUSÕES}

Diante dos objetivos propostos neste estudo, pudemos verificar que, dos 96 graduandos questionados, 90 (93,75\%) conhecem alguma T.A., sendo mais conhecida a terapia floral (citada 58 vezes), seguida da acupuntura (51), homeopatia (43) e cromoterapia (26). Estes achados salientam o fato de que existem diferenças entre as técnicas conhecidas e/ou utilizadas pelos estudantes de Enfermagem, em diferentes países, visto que, enquanto no Brasil a mais conhecida é a terapia floral, no Canadá, segundo Baugniet et al (19), o graduando conhece e faz uso do toque terapêutico, com maior freqüência.

O conhecimento sobre T.A. foi adquirido por intermédio dos veículos de comunicação por $33(34,47 \%)$ graduandos; $26(27,08 \%)$ as conhecem por meio de amigos e familiares e $21(21,87 \%)$ por pessoas que as utilizavam. Podemos concluir que o conhecimento do graduando não é obtido durante a graduação, mas principalmente por intermédio do senso popular.
Com relação ao uso, verificamos que, embora a maioria $(93,75 \%)$ conheça e recomende as T.A. $(86,46 \%)$, apenas poucos graduandos $(36,45 \%)$ as utilizam.

A principal justificativa para a indicação de T.A., segundo os entrevistados, é a crença na sua eficácia e vivência de bons resultados com seu uso. E, embora 92 (95,83\%) dos graduandos afirmem que o enfermeiro pode ser especialista em T.A., apenas 8 $(8,70 \%)$ mostraram conhecimento sobre o caráter legal da especialização para o enfermeiro.

\section{CONSIDERAÇÕES FINAIS}

Podemos notar um certo paradoxo entre o pensar e o agir do estudante, já que muitos recomendam o uso das T.A. baseados apenas no conhecimento da cultura do senso comum, sem nunca ter sequer feito uso de alguma técnica alternativa. Além disso, percebemos que muitos atribuem certa conotação mística às T.A., chegando até mesmo a confundi-las com esoterismo, denotando claramente o desconhecimento sobre os principios e bases científicas de muitas terapias. E, com pesar, observamos que há desconhecimento por parte do graduando sobre as leis que regem o exercício da profissão.

Há ausência de discussões e esclarecimentos sobre o assunto, durante a graduação, na instituição de ensino estudada, já que esta inclui o tema apenas em uma curta disciplina optativa, na qual o número de vagas oferecidas é sempre menor que a demanda de alunos e, assim sendo, nem todos têm a oportunidade de adquirir o conhecimento necessário para a recomendação e uso de técnicas alternativas.

Essa deficiência na formação básica gera uma lacuna no conhecimento do enfermeiro, trazendo prejuizo no seu desempenho profissional, principalmente na assistência à população mais carente, mais afetada pela deficiência do atual Sistema de Saúde. Além disso, a atitude dos estudantes de hoje se reflete no profissional de amanhã e tendências emergentes, no campo da terapêutica alternativa/ complementar, podem trazer implicações para o relacionamento enfermeiro-paciente e para o futuro do cuidado multidisciplinar.

É necessária a urgente intensificação e a oficialização das discussões sobre T.A. em todas as instituições de ensino de Enfermagem, não por meio de cursos extracurriculares ou disciplinas optativas, mas sim como parte do currículo básico do enfermeiro, como disciplina obrigatória e indispensável a todos os graduandos. Afinal, no mundo conturbado em que vivemos, o conhecimento de qualquer técnica que possa auxiliar na assistência à saúde ao indivíduo e que possa aumentar sua qualidade de vida é sempre bem-vindo. 


\section{REFERÊNCIAS BIBLIOGRÁFICAS}

(1) Souza D, Silva MJP. O holismo espiritualista como referencial teórico para o enfermeiro. Rev Esc Enferm USP 1992; $26(2): 235-42$.

(2) Landmann J. As medicinas alternativas: mito, embuste ou ciência ? -- homeopatia, medicina herbal, acupuntura, meditação, ioga, biofeedbach e cura pela fé. Rio de Janeiro: Guanabara; 1989.

(3) Capra F. O ponto de mutação. São Paulo: Cultrix; 1982.

(4) Gerber R. Medicina Vibracional: uma medicina para o futuro. São Paulo: Cultrix; 1988.

(5) Nogueira MJC. Abordagem holística: uma proposta para a enfermagem brasileira. In: Anais do $37^{\circ}$. Congresso Brasileiro de Enfermagem; 1985 nov 17-22; Recife. Recife: Associação Brasileira de Enfermagem; 1986.

(6) Hill A. Guia das medicinas alternativas. São Paulo: Hemus; s.d-

(7) Remen RN. O paciente como ser humano. São Paulo: Summus; 1993.

(8) Silva MJP, Benko MA. O uso da terapias alternativas por enfermeiros docentes. Rev Bras Enferm 1998; 5 (3): 457-68.

(9) Barbosa MA. A utilização de terapias alternativas por enfermeiros brasileiros. [tese] São Paulo (SP):Escola de Enfermagem da USP; 1994.

(10) Organización Mundial de la Salud. Promoción y desarrollo de la medicina tradicional: informe de una reunión de la OMS. Genebra; 1978. (Série Informes Técnicos, 622).

(11) Ernst E. Prevalence of use of complementary/alternative medicine: a systematic review. Bulletin of the World Health Organization 2000; 78 (2): 252-7.

(12) Falcão D. Hospitais se rendem às terapias "alternativas". Folha de São Paulo 2000 maio 11. Suplemento Folha Equilibrio: 10-12.

(13) Price E. The stigma of smell. Nursing Times 1996; 92 (22)..

(14) Nogueira MJC. Terapêuticas alternativas na enfermagem: por que não? Enfoque 1983; 11 (2): 30-35.

(15) Nogueira MJC. Fitoterapia e enfermagem comunitária. [tese] São Paulo (SP): Escola de Enfermagem da USP; 1983.

(16) Calixto JB. Efficacy, safety, quality, control, marketing and regulatory guidelines for herbal medicines (phytotherapeutic agents). Brazilian Journal of Medical and Biological Research 2000; 33 (2): 179-89.

(17) Conselho Federal de Enfermagem. Parecer normativo n. 004/95. Dispõe sobre as atividades em Terapia Alternativa. Bol Inf COREn, v. 18, n.4, p. 8, 1995.
(18) Conselho Federal de Enfermagem. Resolução 197. Estabelece e reconhece as Terapias Alternativas como especialidade e/ou qualificação do profissional de Enfermagem. In: Conselho Regional de Enfermagem. Documentos básicos de enfermagem. São Paulo; 1997

(19) Baugniet J, Boon H, Ostbye T. Complementarary/ Alternative Medicine: comparing the view of medical students in other health care professions. Fam Med 2000; 32 (3): 178-84.

(20) Reed FC, Pettigrew AC, King MO. Alternative and Complementary Therapies in Nursing Curricula. J Nurs Educ 2000; 39 (3): 133-9.

\section{Artigo recebido em 09/02/01}

Artigo aprovado em 19/02/02 